\title{
Effect of dietary fatty acid composition on Th1/Th2 polarization in lymphocytes
}

Takamitsu Mizota, $\mathrm{MD}^{1,2}$; Chiaki Fujita-Kambara, $\mathrm{MD}, \mathrm{PhD}^{2}$; Nemu Matsuya, $\mathrm{MD}^{1,2}$; Shinji Hamasaki, MD, $\mathrm{PhD}^{2}$; Takayasu Fukudome, $\mathrm{MD}, \mathrm{PhD}^{1,2}$; Hirofumi Goto, $\mathrm{MD}, \mathrm{PhD}^{2}$; Shunya Nakane, MD, $\mathrm{PhD}^{2}$; Takayuki Kondo, $\mathrm{MD}, \mathrm{PhD}^{1,2}$; and Hidenori Matsuo, MD, $\mathrm{PhD}^{1,2}$

${ }^{1}$ Department of Clinical Neurosciences, Nagasaki University Graduate School of Biomedical Sciences and ${ }^{2}$ Division of Clinical Research and Department of Neurology, National Hospital Organization, Nagasaki Medical Center of Neurology, Kawatana, Nagasaki 859-3615, Japan

Short title: Dietary fatty acid and Th1/Th2 balance

Key words: polyunsaturated fatty acids, $\alpha$-linolenic acid, T lymphocyte, cytokine, enteral nutrition

Address correspondence: Dr Hidenori Matsuo, Division of Clinical Research and Department of Neurology, National Hospital Organization, Nagasaki Medical Center of Neurology, Shimogumi-gou 2005-1, Kawatana, Higashisonogi-gun, Nagasaki 859-3615, Japan

TEL: +81-956-823121(ext 2003), FAX:+81-956-833710

E-mail: hidenori@kawatana.hosp.go.jp 
Précis $($ limit $=50$ words; words $=41)$

We demonstrated that differences in the composition of n-3 and n-6 polyunsaturated fatty acids, excluding eicosapentaenoic or docosahexaenoic acid, were able to influence the Th1/Th2 balance of lymphocytes in humans as well as in mice, even with normal habitual dietary intake. 
ABSTRACT (limit = 250 words; words $=238$ )

Background: It has become increasingly clear that polyunsaturated fatty acids (PUFAs) have immunomodulatory effects. However, the intake of these fatty acids used in animal studies often is greatly in excess of dietary human intake. Whether differences in the composition of fatty acids that are consumed in amounts consistent with normal habitual dietary intake can influence immune function remains uncertain.

Methods: We manufactured 3 types of liquid diet, related to modified fatty acid composition (n-6/n-3 = 0.25, 2.27 and 42.9), but excluding eicosapentaenoic acid and docosahexaenoic acid, based upon a liquid diet used clinically in humans. We assessed CD3-stimulated cytokine production of splenocytes in female BALB/c mice ( $n=4$ per group) fed one of three liquid diets for $4 \mathrm{w}$. We also measured the cytokine production of peripheral blood mononuclear cells stimulated with phorbol myristate acetate and ionomycin in humans at the end of a 4-week period of consumption of two different liquid diets (n-6/n-3 = 3 and 44). Results: We found that the ratio of interferon- $\gamma$ (IFN- $\gamma$ ) / interleukin-4 (IL-4) was significantly higher in mice fed the n-3 rich diet than in others. In humans, IFN- $\gamma$ / IL-4 was significantly higher after the n-3 versus the n-6 enhanced diet.

Conclusions: Differences in the composition of n-3 and n-6 PUFAs induces a shift in the Th1/Th2 balance in both mouse and human lymphocytes, even when ingested in normal dietary amounts. An n-3 rich diet containing $\alpha$-linolenic acid modulates immune function. 


\section{BACKGROUND}

Environmental factors in the Western lifestyle probably contribute to the development of atopy and allergies. A decrease in saturated fatty acid consumption and an increase in polyunsaturated fatty acid (PUFA) consumption in the diet have been linked to the increased prevalence of atopic diseases in industrialized countries ${ }^{1}$. PUFAs are classified as $n-3$ or $n-6$, on the basis of the location of the last double bond relative to the terminal methyl ( $\omega$ ) end of the molecule. The intake of PUFAs results in uptake into essentially every cell of the body, where they bind fatty acid-binding proteins and undergo metabolic conversion ${ }^{2}$. There are 2 PUFAs: linoleic acid (LA, 18:2 n-6) and $\alpha$-linolenic (ALA, 18:3 n-3), which cannot be synthesized de novo by human cells and, hence, have been designated as essential fatty acids. These essential fatty acids undergo a series of saturation, de-saturation, and elongation steps that can result in the formation of long-chain PUFAs. Common dietary sources of n-6 PUFAs are corn, safflower, soybean, and sunflower oils ${ }^{3}$. LA is converted to arachidonic acid (AA, 20:4 n-6) which, along with other PUFAs (including some $n-3$ fatty acids), can serve as the precursor for the eicosanoid mediators of inflammation (prostaglandins, thromboxanes, and leukotrienes). ALA, which is obtained from green leafy vegetables, walnuts, and rapeseed and flaxseed oils, can be further elongated, although not very efficiently, to long-chain eicosapentaenoic acid (EPA, 20:5 n-3) ${ }^{4}$. Most of the long chain n-3 PUFAs, EPA, and docosahexaenoic acid (DHA, 22:6 n-3) are obtained directly from the dietary intake of marine fish oils. Typical Western diets contain almost 10 times more LA (n-6) than ALA (n-3) ${ }^{5}$. In Japan, the prevalence of atopic diseases has been rising, which has been paralleled by a decrease in the ratio of n-3 to n-6 PUFA consumption. Conversely, epidemiologic studies in a 
population of coastal Eskimos have revealed that a high intake of n-3 PUFAs correlates with a low incidence of cardiovascular and inflammatory diseases, like asthma and type I diabetes mellitus ${ }^{6}$.

It has become increasingly clear that PUFAs have immune-modulating effects in cell culture and in vivo ${ }^{7-10}$. PUFAs can modulate cytokine production, lymphocyte proliferation, surface molecule expression, phagocytosis, and apoptosis, and they can inhibit natural killer cell activity ${ }^{11-19}$. In general, n-3 PUFAs are considered good for human health, whereas n-6 PUFAs are deemed less beneficial or even harmful ${ }^{3}$. Although studies are not conclusive and there have been conflicting reports ${ }^{20}$, the $n-3$ PUFAs appear to decrease specific disease symptoms and the need for anti-inflammatory drugs among patients with chronic inflammatory diseases ${ }^{7,21}$. In addition, $\mathrm{n}-3$ fatty acids may act as immunosuppressive agents for a variety of inflammatory disorders, including Crohn's disease, atherosclerosis, colitis, graft-versus-host disease, rheumatoid arthritis, psoriasis, multiple sclerosis, asthma, and systemic lupus erythematosus ${ }^{22-31}$.

Animal feeding studies have indicated that a high intake of n-6 and n-3 fatty acid can influence lymphocyte functions and cell-mediated immunity. The impact of dietary fatty acids on animal autoimmune disease models appears to depend upon the animal model and the type and amount of fatty acid that the animals are fed $^{32}$. The weight-relative intake of these fatty acids used in animal studies often is greatly in excess of that achievable in humans ${ }^{33}$. Consequently, the results of studies involving human autoimmune disease have been less dramatic, even though human trials have been subject to uncontrolled dietary and genetic backgrounds, infection and other environmental influences, and their basic trial designs have 
been inadequate ${ }^{32}$. Nonetheless, large long-term differences in the habitual intake of these fatty acids might influence immune function.

Recently, many types of enteral nutrient solutions have been developed that are being used for chronically ill patients, as a long-term source of nutrition. There is considerable variety in the fatty acid composition of these liquid diets; some contain a large amount of n-6 PUFAs, and others more n-3. In this two-part study, we first investigated the effects of dietary fatty acid on T-lymphocyte polarization in mice using three such liquid diets, all the same composition except for fatty acid content. Second, we examined whether differences in the fatty acid composition of two otherwise similar (but not identical) liquid diets affect the Th1/Th2 polarization of lymphocytes in human patients receiving long-term enteral nutrition.

\section{MATERIALS AND METHODS}

Animal experiments

To determine whether the composition of fatty acids in a habitual diet affects Th1/Th2 polarization, we fed three separate groups of female BALB/c mice ( $n=4$ per group) one of three different liquid diets for 4 weeks. These diets were manufactured by modifying the fatty acid composition of a liquid diet used clinically for patients, without changing the composition of any other component, including its nutrients, electrolytes, calories $(1 \mathrm{kcal} / \mathrm{ml})$ and vitamins (Table 1). Each liquid diets contained 1.96g/100kcal oil. In addition, Diet A contained perilla seed oil ( $n-6 / n-3=0.25$, n-3 rich); Diet B contained 28\% perilla seed oil and 72\% corn oil (n-6/n-3= 2.27, n-3 enhanced) and Diet $C$ contained corn oil (n-6/n-3= 42.9, n-6 rich) (Table 2). 
After 4 weeks, mononuclear cells were isolated from the spleen of each mouse. 96-well round-bottomed micro-culture plates were coated with $10 \mu \mathrm{g} / \mathrm{ml}$ of purified anti-mouse CD3e monoclonal Ab (Cedarlane, Ontario Canada), followed by three washes with Hanks solution. Cells were cultured (at a concentration of $2 \times 10^{6} / \mathrm{ml}$ ) in quadruplicate cultures at $37^{\circ} \mathrm{C}$ in $5 \% \mathrm{CO}_{2}$ in RPMI 1640 medium containing antibiotics, $1 \mathrm{mM}$ L-glutamine, $5 \times 10^{-5} \mathrm{M}$ 2-mercaptoethanol (ME), and 10\% FCS , for 24h. The production of interferon- $\gamma$ (IFN- $\gamma$ ) and interleukin-4 (IL-4) in the culture supernatant was measured by ELISA in triplicate, using an AN'ALYZA ${ }^{\mathrm{TM}}$ Immunoassay System (mouse IFN- $\gamma$ and IL-4) supplied by TECHNE Co. (Minneapolis, MN55413, USA).

Animal experiments were conducted in accordance with the guidelines of the Animal Care and Use Committee of Nagasaki University School of Medicine. During the observation period, mice were housed under standard, approved conditions.

\section{Clinical study}

Ten patients with neurodegenerative disorders or in the chronic stages of a cerebrovascular accident (CVA) who were being fed exclusively through an enteral tube were enrolled in this study (Table 3). Fully informed consent was obtained from the each patient or from an appropriate family member. The institutional review board had approved the project prior to subject recruitment.

Using a cross-over design composed of two sequential four-week observation periods, we gave each patient enteral feeding formula RAC (Racol; n-6/n-3 = 3, EN Otsuka, Japan) for 
4 weeks, either preceded or followed by four weeks of EL (Ensure Liquid; n-6/n-3 = 44, Abbott Japan Co., Ltd.). The RAC formula contained a combination of perilla, soybean, palm oil and medium-chain triglyceride as its source of lipids; meanwhile, among these four fatty acid ingredients, EL contained corn oil exclusively (Table4). Both formulae contained ALA, but not EPA or DHA. For the duration of each 4-week period, water intake and total calories (1200 to1600 kcal) remained unchanged for any given patient, and no other nutrition was given.

Blood samples were taken at the end of each 4-week observation period. Peripheral blood mononuclear cells (PBMCs) were isolated from whole blood using density gradient centrifugation, and then washed twice with Hanks' balanced salt solution. PBMCs ( 2 x $10^{5}$ /well) were cultured in RPMI 1640 medium containing 10\% FBS, antibiotics, and Lglutamine, with phorbol myristate acetate (PMA: 20ng/ml) and ionomycin (500ng/ml) in $200 \mu \mathrm{l}$ in 96 -well flat-bottomed plates. After $24 \mathrm{hrs}$ of culture at $37^{\circ} \mathrm{C}$ in $5 \% \mathrm{CO}_{2}$, the culture supernatant was removed for cytokine assay. The production of IFN- $\gamma$ and IL-4 in the culture supernatant was measured by ELISA in triplicate, using an AN'ALYZA ${ }^{\mathrm{TM}}$ Immunoassay System (Human IFN- $\gamma$ and IL-4) supplied by TECHNE Co. (Minneapolis, MN55413, USA).

\section{Statistical analysis}

All values were calculated as means \pm SD. The results of cytokine assays were done in triplicate with SDs of $<10 \%$. Inter-group differences in the animal experiments were tested for statistical significance using Fisher's PLSD test, and those in the clinical study by 
Wilcoxon rank sign test. A difference of $p<0.05$ was considered statistically significant, and all tests were two-tailed.

\section{RESULTS}

Animal experiments

In the animal experiment, there was no significant inter-group difference in body weight between the 3 groups of mice at the end of the feeding: group A (fed Diet A, n-3 rich diet) weighed $22.8 \pm 1.0$ g; group B( fed Diet B, n-3 enhanced diet) $23.6 \pm 2.1$ g; group C (fed Diet C, n-6 rich diet) $23.5 \pm 2.0 \mathrm{~g}$.

Production of IFN $\gamma$ by anti-CD3-stimulated splenocytes declined significantly in the mice fed the n-6 rich diet (Diet C) relative to that observed in the mice fed n-3 rich Diet A (Fig. 1a; $\mathrm{p}=0.0097$ ). Production of IL-4 by splenocytes in mice fed the $\mathrm{n}-3$ rich Diet $\mathrm{A}$ or the n-6 rich Diet C was significantly reduced relative to mice fed Diet B (Fig. 1b; $p=0.0044$ and 0.0245, respectively). As a result, the ratio of IFN- $\gamma$ to IL-4 was significantly higher in the mice fed n-3 rich Diet A than in mice fed either of the other two diets ( Fig. 1c; $p=0.0226$ and 0.0235, respectively).

\section{Clinical study}

During the 8 weeks of observation, 4 patients developed fever or some other inflammatory sign(s) and were excluded from the study. We ultimately analyzed the production of IFN- $\gamma$ and IL-4 by PBMCs from only 6 patients who had had 4 weeks of consumption of both the n-3 enhanced diet and the n-6 rich diet, in the absence of fever or 
other signs of inflammation. The clinical characteristics of these 6 patients are shown in Table 3. Three patients were fed the n-3 enhanced diet (RAC) during the first 4 week period, before crossing over to the alternative n-6 rich diet (EL); and the other 3 patients received the same two diets, but in the reverse order.

The production of IFN- $\gamma$ by PBMCs stimulated with PMA and ionomycin increased in 2 and decreased in 4 patients during their four weeks being fed the n-6 rich diet (Fig. 2). The production of IL-4 by PBMCs rose more in every patient while on the n-6 rich diet than on the n-3 enhanced diet (Fig. 2; $p=0.0277$ ). As a result, the ratio of IFN- $\gamma$ to IL-4, which may be a better index of actual Th1/Th2 cytokine-production, was significantly higher during consumption of the $n-3$ versus the $n-6$ rich diet (Fig. $2 ; p=0.0277$ ).

\section{DISCUSSION}

In the present study, we demonstrated that liquid diets with different compositions of n3 and n-6 PUFAs are able to induce a shift in the Th1/Th2 balance of lymphocytes in both humans and mice, even when administered in normal dietary amounts. The fact that an n-3 rich diet containing ALA, in the absence of DHA and EPA (marine fish oil), still modulated immune function is another important finding.

In vitro and animal feeding studies have suggested that PUFAs have the capacity to modulate lymphocyte functions and cell-mediated and antibody responses to various challenges. However, the quantities of fatty acid administered to animals in these studies often were greatly in excess of the relative dietary intake of humans. In our own animal experiment, we fed mice one of 3 different liquid diets, different only in their fatty acid content. Because 
these liquid diets were formed based on the composition of a liquid diet (RAC) commonly used clinically in humans, the amount of fatty acid was not excessive for mice. Nevertheless, the ratios of Th1 to Th2 cytokine production were strikingly different between the 3 groups of mice.

Although previous animal studies have demonstrated that diets rich in n-3 PUFA can exert anti-inflammatory and immunomodulatory effects in vivo, there have been relatively few good studies in humans. In the present clinical study, we used 2 different liquid diets that are clinically available in Japan. Accordingly, the two fluid supplements were different in several nutrients other than fatty acids; however, these differences are minimal (Table 4) because both liquid diets are formulated to satisfy well-established daily nutrient requirements. Moreover, the differences in the other nutrients paled next to the sizeable differences in fatty acid content, so that we believe that it was the difference in fatty acid composition that induced the change of Th1/ Th2 balance of lymphocytes we observed. Admittedly, we could be wrong; it is possible that the small differences in vitamin and trace mineral content could have influenced immune function, but we think that unlikely.

Other investigators have speculated that the ratio of $n-3$ to $n-6$ may be an important factor in the modulation of inflammation and autoimmunity ${ }^{34}$. Increasing the ratio of $n-3$ to n6 relieves symptoms associated with some inflammatory diseases ${ }^{35}$, although this may not hold true in all cases ${ }^{36}$.

Our observation seems to be accordance with previous animal studies, which have generated considerable evidence that feeding animals either plant or fish oils rich in n-3 PUFAs does alter the ex vivo production of cytokines, like tumor necrosis factor (TNF), 
interleukin 1 (IL-1), interleukin 6(IL-6 )and interleukin 2(IL-2). The n-6 PUFA, arachidonic acid, gives rise to the eicosanoid family of inflammatory mediators (prostaglandins, leukotrienes and related metabolites), whereas n-3 PUFAs act as arachidonic acid antagonists. However, it remains to be seen which mechanism(s) led to the immunomodulation observed in the present study. We did not investigate which fatty acid contributed to the change in Th1/Th2 ratio or which immune cells were central to that process.

In the present study, each liquid diet contained ALA as the only source of n-3 fatty acids, albeit at different doses. It is believed that ALA has immunomodulatory effects that are similar to those of EPA and DHA, although its effect seems to be much weaker. It is not clear how EPA, DHA and other metabolites contributed to the present results, because we did not investigate to what extent ALA was elongated in vivo. Only a few studies have investigated the immunologic effects of the precursor, n-3 PUFA ALA, in humans. Caughey et al ${ }^{37}$ reported that $13.7 \mathrm{~g}$ ALA / $\mathrm{d}$ for 4 weeks results in a decrease in production of TNF- $\alpha$ and IL$1 \beta$ by endotoxin-stimulated mononuclear cells of $27 \%$ and $30 \%$, respectively. By comparison, fish oil providing $2.7 \mathrm{~g}$ EPA+DHA/d decreased production of these cytokines by $70 \%$ and $78 \%$, respectively ${ }^{37}$. Given that roughly one fifth the weight of EPA+DHA produced approximately twice the decline in cytokine production as ALA, long-chain n-3 PUFAs appear to be about 9-10 times as potent as ALA with respect to the inhibition of cytokine production in healthy subjects. In contrast, several studies using lower intakes of ALA (2-9.5 g/d) did not identify effects on TNF- $\alpha$, IL-1 $\beta$, or IL-6 production by endotoxin-stimulated mononuclear cells ${ }^{38-40}$ or on IL-2, IL-4, or IFN $\gamma$ production by mitogen-stimulated mononuclear cells ${ }^{39}$. These data suggest that increasing ALA intake to $>10 \mathrm{~g} / \mathrm{d}$ is required for 
anti-inflammatory or immunomodulatory effects to be seen. Even then, the effects will be much more modest than those exerted by long-chain n-3 PUFAs ${ }^{37}$. In fact, only Diet A, which contained a large amount of ALA, appeared to suppress the Th2 response and enhance the Th1 response in the current animal study. Meanwhile, Diet B, corresponding to RAC, contributed less to IFN $\gamma$ production.

Of note is that our patients received, at most, only 1.8-2.4g ALA/d during the 4-week period in which they were fed the $n-3$ enhanced diet. Nevertheless, the Th1/Th2 balance in PBMCs shifted to Th1, even though we only assessed TCR-stimulated cytokine production. Apparently, the present clinical study differs from previous ones in several respects, which include the total fat amount, subject characteristics, and methods of immunologic evaluation. In particular, our subjects were not healthy; rather, they were chronically ill patients with a neurological disorder, which itself may affect lymphocyte function. In addition, all patients depended on long-term enteral nutrition, which may have altered ALA metabolism. Tanaka et al. ${ }^{41}$ reported that the same liquid diet (RAC) used in the present study induce significant increases in the serum concentration of EPA, DPA and DHA, as well as ALA. Their patients also had severe motor and intellectual disabilities and relied on long-term enteral nutrition for all of their nutritional needs.

Albeit preliminary, our findings suggests that liquid diets rich in n-3 PUFAs can be of use in the treatment of immunological or inflammatory disorders. Human trials, designed to control for dietary and genetic background and other environmental influences, clearly are warranted to formally assess the therapeutic potential of n-3 rich diets. 


\section{Acknowledgement}

We are grateful to Kenichi Ogasawara and Seishi Tanimoto for the precious advice and the thoughtful encouragement. This work was supported in part by the Health and Labour Sciences Research Grant on Intractable Diseases (Neuroimmunological Diseases) from the Ministry of Health, Labour and Welfare of Japan. None of the authors had any financial or personal interest in any company or organization. 


\section{References}

1. Kankaanpää P, Süta Y, Salminen S, Lichtenstein A, Isolauri E. Diatary fatty acid and allergy. Ann Med. 1999, 31:282-287.

2. Jump DB. The biochemistry of n-3 polyunsaturated fatty acids. J Biol Chem. 2002;277:8755-8758.

3. Siddiqui RA, Shaikh SR, Sech LA, Yount HR, Stillwell W, Zaloga GP. Omega 3-fatty acids: health benefits and cellular mechanisms of action. Mini Rev Med Chem. 2004;4:859-871.

4. Burdge GC, Calder PC. Conversion of alpha-linolenic acid to longer-chain linolenic acid to longer-chain polyunsaturated fatty acids in human adults. Reprod Nutr Dev. 2005;45:581-97.

5. Calder PC. Fat chance of immunomodulation. Immnol Today. 1998, 246:244-247

6. Kromann N, Green HO. Epidemiological studies in the Upernavic district, Greenland. Acta Med Scand.1986, 208:401-406.

7. Simopoulos AP. Omega-3 fatty acids in inflammation and autoimmune diseases. $J$ Am Coll Nutr. 2002;21:495-505.

8. Shaikh SR and Edidin M. Polyunsatulated fatty acids, membrane organization,T cells, and antigen presentation. Am J Clin Nutr. 2006;84:1277-1289

9. Calder PC. n-3 Polyunsatulated fatty acids, inflammation, and inflammatory disease. Am $J$ Clin Nutr. 2006;83(suppl):1505S-1519S

10. Stulnig TM. Immunomodulation by polyunsaturated fatty acids: mechanisms and effects. Int Arch Allergy Immunol. 2003;132:310-321. 
11. Costabile M, Hii CST, Melino M, Easton C, Ferrante A. The immunomodulatory effects of novel ß-oxa, ß-thia, and $\gamma$-thia polyunsaturated fatty acids on human $\mathrm{T}$ lymphocyte proliferation, cytokine production, and activation of protein kinase C and MAPKs. $J$ Immunol. 2005;174:233-243.

12. Lokesh BR, Sayers TJ, Kinsella JE. Interleukin-1 and tumor necrosis factor synthesis by mouse peritoneal macrophages is enhanced by dietary n-3 polyunsaturated fatty acids. Immunol Lett.1990;23:281-285.

13. Hughes DA, Southon S, Pinder AC. (n-3) Polyunsaturated fatty acids modulate the expression of functionally associated molecules on human monocytes in vitro. $J$ Nutr. 1996;126:603-10.

14. Calder PC, Bond JA, Harvey DJ, Gordon S, Newsholme EA. Uptake and incorporation of saturated and unsaturated fatty acids into macrophage lipids and their effect upon macrophage adhesion and phagocytosis. Biochem J. 1990;269:807-814.

15. Kew S, Gibbons ES, Thies F, McNeill GP, Quinlan PT, Calder PC. The effect of feeding structured triacylglycerols enriched in eicosapentaenoic or docosahexaenoic acids on murine splenocyte fatty acid composition and leucocyte phagocytosis. Br J Nutr. 2003;90:1071-1080.

16. Finstad HS, Dyrendal H, Myhrstad MCW, Heimli H, Drevon CA. Uptake and activation of eicosapentaenoic acid are related to accumulation of triacylglycerol in Ramos cells dying from apoptosis. J Lipid Res. 2000;41:554-563. 
17. Meydani SN, Yoogeswaran G, Liu S, Baskar S, Meydani M. Fish oil and tocopherolinduced changes in natural killer cell-mediated cytotoxicity and PGE2 synthesis in young and old mice. $J$ Nutr. 1988;1245-1252.

18. Calviello G, Palozza P, Piccioni E, et al. Dietary supplementation with eicosapentaenoic and docosahexaenoic acid inhibits growth of Morris hepatocarcinoma 3924A in rats: effects on proliferation and apoptosis. Int J Cancer. 1998;75:699-705.

19. Calder PC, Bevan SJ, Newsholme EA. The inhibition of T-lymphocyte proliferation by fatty acids is via an eicosanoid-independent mechanism. Immunology. 1992;75:108-115.

20. Michalsen A, Lehmann N, Pithan C, et al. Mediterranean diet has no effect on markers of inflammation and metabolic risk factors in patients with coronary artery disease. Eur $J$ Clin Nutr. 2006;60:478-485.

21. Calder PC. Dietary modification of inflammation with lipids. Proc Nutr Soc. 2002;61:345-358.

22. Thies F, Garry JM, Yaqoob P, et al. Association of n-3 polyunsaturated fatty acids with stability of atherosclerotic plaques: a randomised controlled trial. Lancet. 2003;361:477_ 485.

23. Mills SC, Windsor AC, Knight SC. The potential interactions between polyunsaturated fatty acids and colonic inflammatory processes. Clin Exp Immunol. 2005;142:216-228.

24. Dwyer JH, Allayee H, Dwyer KM, et al. Arachidonate 5-lipoxygenase promoter genotype, dietary arachidonic acid, and atherosclerosis. N Engl J Med. 2004;350:29-37. 
25. Takatsuka H, Takemoto Y, Yamada S, et al. Oral eicosapentaenoic acid for acute colonic graft-versus-host disease after bone marrow transplantation. Drugs Exp Clin Res. 2002;28:121-125.

26. Camuesco D, Galvez J, Nieto A, et al. Dietary olive oil supplemented with fish oil, rich in EPA and DHA (n-3) polyunsaturated fatty acids, attenuates colonic inflammation in rats with DSS-induced colitis. J Nutr. 2005;135:687-694.

27. Belluzzi A, Brignola C, Campieri M, Pera A, Boschi S, Miglioli M. Effect of an entericcoated fish-oil preparation on relapses in Crohn's disease. N Engl J Med. 1996;334:15571560.

28. Mayser P, Grimm H, Grimminger F. n-3 Fatty acids in psoriasis. Br J Nutr. 2002;87(suppl):S77-S82.

29. Gallai V, Sarchielli P, Trequattrini A, et al. Cytokine secretion and eicosanoid production in the peripheral blood mononuclear cells of MS patients undergoing dietary supplementation with n-3 polyunsaturated fatty acids. J Neuroimmunol. 1995;56:143153.

30. Broughton KS, Johnson CS, Pace BK, Liebman M, Kleppinger KM. Reduced asthma symptoms with n-3 fatty acid ingestion are related to 5-series leukotriene production. Am J Clin Nutr.1997;65:1011-1017.

31. Arita M, Yoshida M, Hong S, et al. Resolvin E1, an endogenous lipid mediator derived from omega-3 eicosapentaenoic acid, protects against 2,4,6-trinitrobenzene sulfonic acidinduced colitis. Proc Natl Acad Sci U S A. 2005;102:7671-7676. 
32. Harbige LS.Dietary n-6 and n-3 fatty acids in immunity and autoimmune disease.Proc Nutr Soc. 1998;57:555-562.

33. Calder PC, Yaqoob P, Thies F, Wallace FA, Miles EA. Fatty acids and lymphocyte functions. Br J Nutr. 2002;87(Suppl 1): S31-S48.

34. Simopoulos AP. Importance of the ratio of omega-6/omega-3 essential fatty acids: evolutionary aspects. World Rev Nutr Diet. 2003;92:1-22

35. Broughton KS, Johnson CS, Pace BK, Liebman M, Kleppinger KM. Reduced asthma symptoms with n-3 fatty acid ingestion are related to 5-series leukotriene production. Am J Clin Nutr. 1997;65:1011-1017

36. Calder PC, Miles EA. Fatty acids and atopic disease. Pediatr Allergy Immunol. 2000;11(suppl):29-36.

37. Caughey GE, Mantzioris E, Gibson RA, Cleland LG, James MJ. The effect on human tumor necrosis factor $\alpha$ and interleukin $1 ß$ production of diets enriched in $n-3$ fatty acids from vegetable oil or fish oil. Am J Clin Nutr. 1996;63:116-122.

38. Thies F, Miles EA, Nebe-von-Caron G, et al. Influence of dietary supplementation with long chain n-3 or n-6 polyunsaturated fatty acids on blood inflammatory cell populations and functions and on plasma soluble adhesion molecules in healthy adults. Lipids. 2001;36:1183-1193.

39. Kew S, Banerjee T, Minihane AM, et al. Lack of effect of foods enriched with plant- or marine-derived n-3 fatty acids on human immune function. Am J Clin Nutr. 2003;77:1287-1295. 
40. Wallace FA, Miles EA, Calder PC. Comparison of the effects of linseed oil and different doses of fish oil on mononuclear cell function in healthy human subjects. Br J Nutr. 2003;89:679-689

41. Tanaka Y, Mizote H, Inada H, et al. Efficacy of n-3 polyunsaturated fatty acid enriched enteral nutrient solution in relieving oxidative stress in patients with severe psychophysiologic disorders. Kurume Med J. 2004;51:83-90 
Table 1. Composition of the liquid diets for the animal experiment

\begin{tabular}{|c|c|c|c|c|}
\hline Ingredients & & Diet A & Diet B & Diet C \\
\hline Perilla oil & $(\mathrm{g} / 100 \mathrm{ml})$ & 1.96 & 0.50 & - \\
\hline Corn oil & & - & 1.46 & 1.96 \\
\hline Casein & $(\mathrm{g} / 100 \mathrm{ml})$ & \multicolumn{3}{|c|}{3.40} \\
\hline Soybean protein isolated & & \multicolumn{3}{|c|}{1.67} \\
\hline Dextrin & & \multicolumn{3}{|c|}{14.86} \\
\hline Sucrose & & \multicolumn{3}{|c|}{1.30} \\
\hline Emulsifier* & & \multicolumn{3}{|c|}{0.30} \\
\hline Minerals & $(/ 100 \mathrm{ml})$ & \multicolumn{3}{|c|}{ 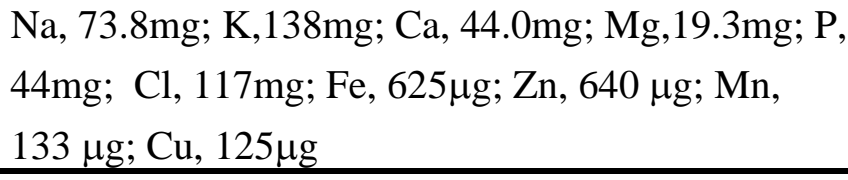 } \\
\hline Vitamines & $(/ 100 \mathrm{ml})$ & \multicolumn{3}{|c|}{ 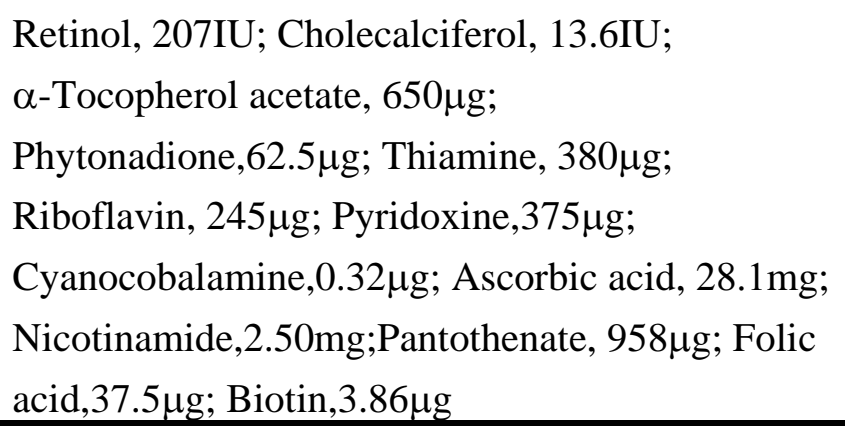 } \\
\hline Total energy & $\begin{array}{l}\text { (kcal/100ml } \\
\text { ) }\end{array}$ & 100 & 100 & 100 \\
\hline Osmotic pressure & $(\mathrm{mOsm} / \mathrm{L})$ & 400 & 399 & 403 \\
\hline
\end{tabular}


Table 2. Fatty acid composition of the experimental liquid diets.

\begin{tabular}{lllll}
\hline & & Diet A & Diet B & Diet C \\
\hline C8:0 & $(\%)$ & 36.9 & 38.1 & 38.2 \\
C16:0 (Palmitic acid) & $(\%)$ & 5.3 & 7.6 & 8.5 \\
\hline C18:0 (Stearic acid) & $(\%)$ & 1.9 & 2.1 & 2.2 \\
C18:1 (Oleic acid) & $(\%)$ & 9.5 & 17.8 & 20.2 \\
C18:2 (Linoleic acid) & $(\%)$ & 9.4 & 24.1 & 30.1 \\
C18:3 ( $\alpha$-Linolenic acid) $(\%)$ & 37.0 & 10.6 & 0.7 \\
\hline n-6 / n-3 & & 0.25 & 2.27 & 42.9 \\
\hline n-3 + n-6 & $(\%)$ & 46.4 & 34.7 & 30.8 \\
\hline
\end{tabular}


Table 3. Characteristics of the subjects.

\begin{tabular}{lllll}
\hline Patient & Sex & Age & Disease & $\mathbf{1}^{\text {st }} \mathbf{4}$ wk $/ \mathbf{2}^{\text {nd }} \mathbf{4 w k}$ \\
\hline 1 & F & 89 & Cerebral hemorrhage & EL / RAC \\
2 & F & 75 & Multi-system atrophy & EL / RAC \\
3 & F & 55 & Parkinson's diseaese & EL / RAC \\
4 & F & 75 & Corticobasal degeneration & RAC / EL \\
5 & F & 83 & Parkinson's diseaese & RAC / EL \\
6 & M & 77 & Huntington chorea & RAC / EL \\
\hline
\end{tabular}


Table 4. Composition of the diets for the clinical study (per $100 \mathrm{~mL}$ )

\begin{tabular}{|c|c|c|}
\hline Component & Racol (RAC) & Ensure Liquid (EL) \\
\hline Energy(kcal) & 100 & 100 \\
\hline (protein / fat / carbohydrate ) & $(18 / 20 / 62)$ & $(14.1 / 31.5 / 54.5)$ \\
\hline \multicolumn{3}{|l|}{ Protein } \\
\hline Casein protein (g) & 3.4 & 3.4 \\
\hline Soy protein (g) & 1.7 & 0.5 \\
\hline \multicolumn{3}{|l|}{ Fat } \\
\hline Medium-chain triglyceride (mg) & 750 & 0 \\
\hline Myrstic acid (mg) & 0 & 14.1 \\
\hline Palmitic acid (mg) & 269 & 415.4 \\
\hline Stearic acid (mg) & 72 & 84.5 \\
\hline Oleic acid (mg) & 342 & 929.3 \\
\hline Linoleic acid (mg) & 450 & 1992.3 \\
\hline$\alpha$-Linolenic acid (mg) & 150 & 45.8 \\
\hline Arachidonic acid (mg) & 0 & 21.1 \\
\hline n-6 fatty acid (mg) & 450 & 2013.4 \\
\hline n-3 fatty acid (mg) & 150 & 45.8 \\
\hline$n-6 / n-3$ & 3 & 44 \\
\hline Carbohydrate (g) & 15.6 & 13.7 \\
\hline \multicolumn{3}{|l|}{ Vitamins and trace elements } \\
\hline Vitamin A (IU) & 207 & 250 \\
\hline Vitamin E (mg) & 0.7 & 3 \\
\hline Vitamin C (mg) & 28.1 & 15.2 \\
\hline Folate $(\mu \mathrm{g})$ & 37.5 & 20 \\
\hline Vitamin B6 (mg) & 0.4 & 0.2 \\
\hline Vitamin B12 $(\mu \mathrm{g})$ & 0.3 & 0.6 \\
\hline $\mathrm{Fe}(\mathrm{mg})$ & 0.6 & 0.9 \\
\hline $\mathrm{Cu}$ (mg) & 0.1 & 0.1 \\
\hline Mn (mg) & 0.1 & 0.2 \\
\hline $\mathrm{Zn} \mathrm{(mg)}$ & 0.6 & 1.5 \\
\hline
\end{tabular}




\section{Figure legends}

Fig.1 Production of cytokines of splenocytes stimulated with anti-CD3 in the mice fed, n-3 rich $\operatorname{diet}($ Diet A) , n-3 enhanced diet (Diet B), or n-6 rich diet (Diet C). a; production of interferon- $\gamma$ ( IFN- $\gamma$ ), b; production of interleukin-4(IL-4), c; ratio of IFN- $\gamma /$ IL-4. The results of cytokine assays were in triplicate with SDs of $<10 \%$. $*$ signifies $p<0.05$ (Fisher's PLSD test).

Fig.2 Production of cytokines of peripheral blood mononuclear cells stimulated with phorbol myristate acetate and ionomycin in the patients at the end of each 4-week observation period of feeding with n-3 enhanced diet (Racol) and n-6 rich diet(Ensure Liquid). a; production of interferon- $\gamma$ ( IFN- $\gamma$ ), b; production of interleukin-4(IL-4), c; ratio of IFN- $\gamma /$ IL-4. The results of cytokine assays were in triplicate with SDs of $<10 \%$. * signifies $p<0.05$ (Wilcoxon rank sign test). Pt No. is corresponding to that in Table 3. 


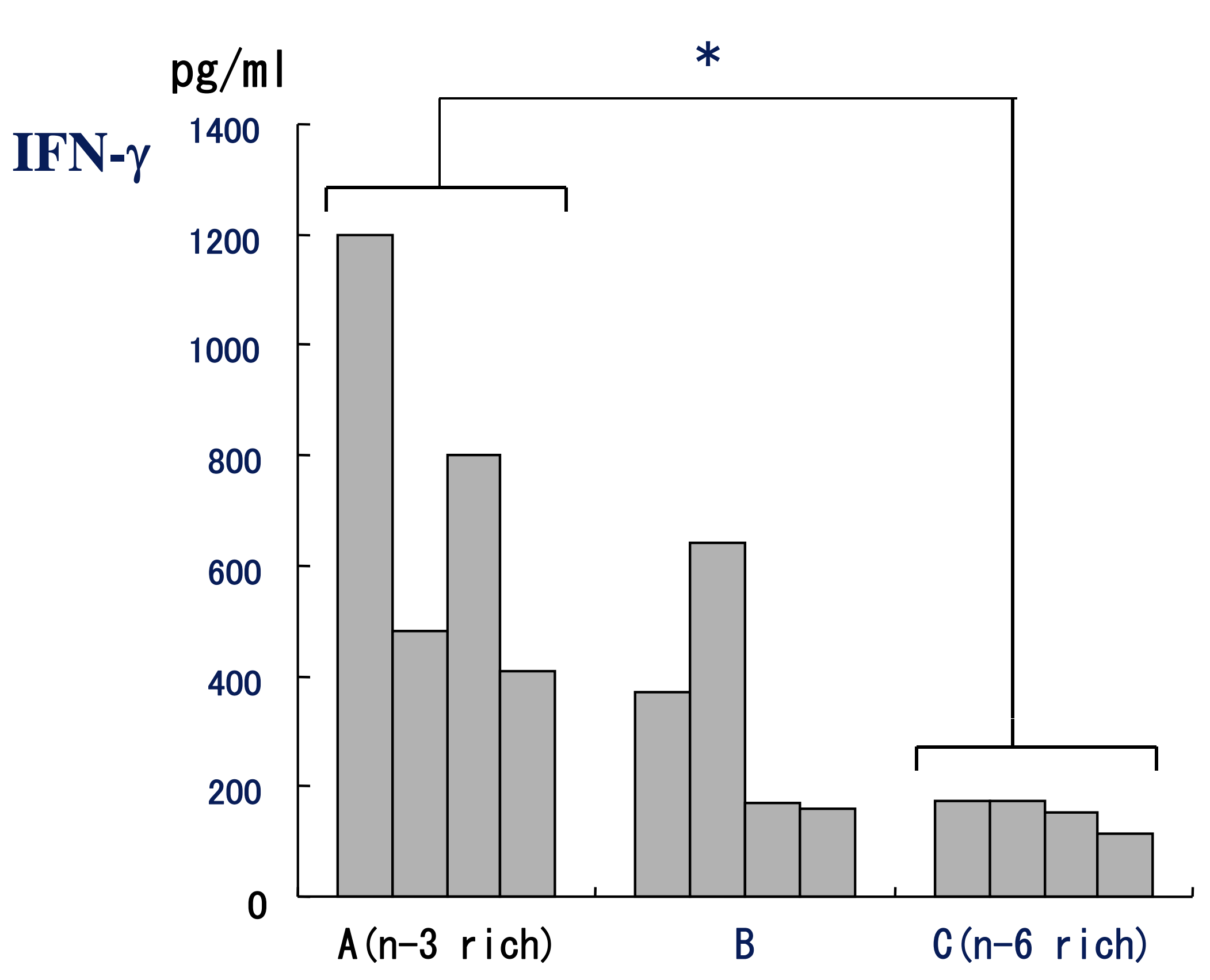

Fig.1a 


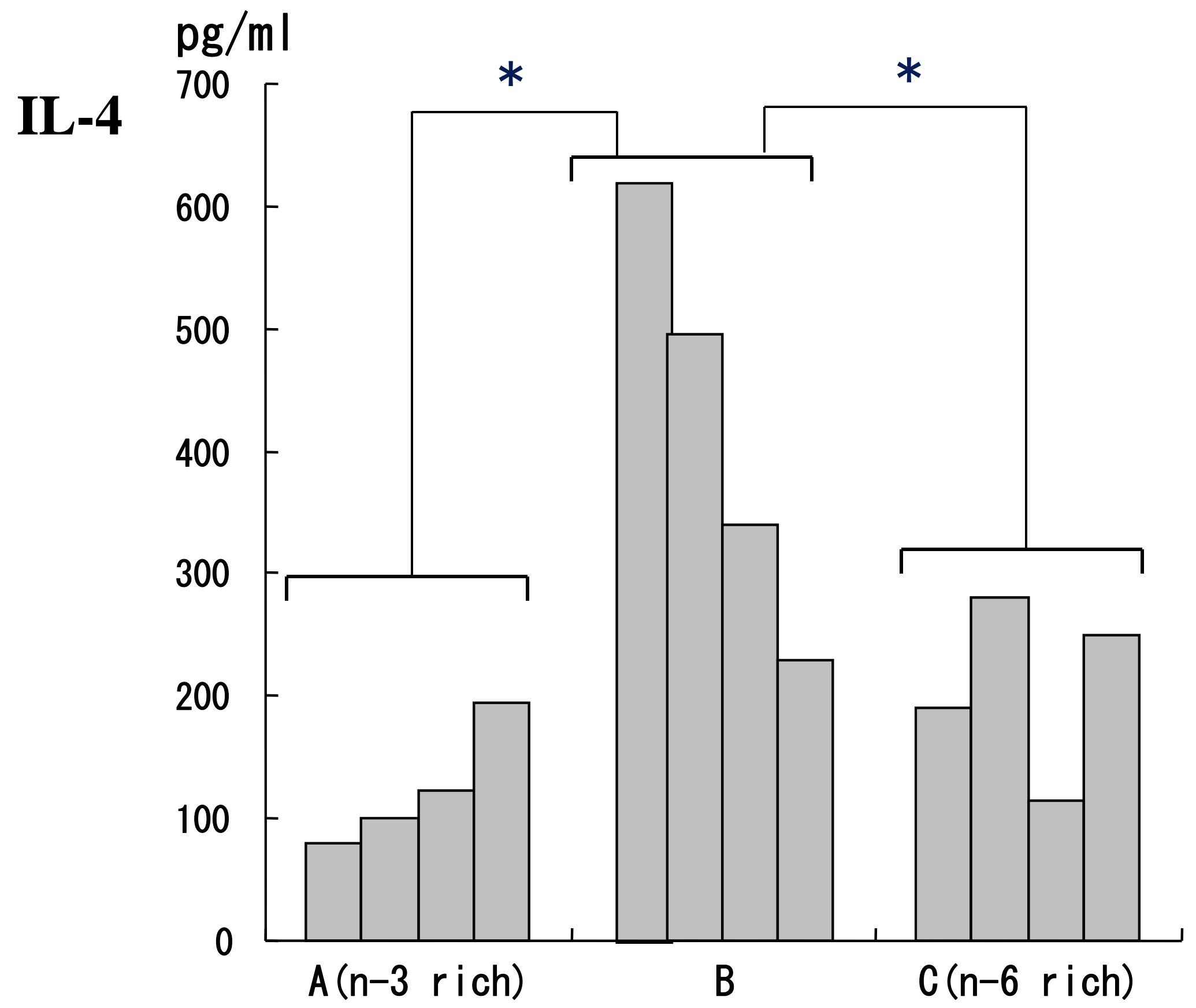

Fig. 1b 
IFN- $\gamma /$ IL-4

Fig. 1c

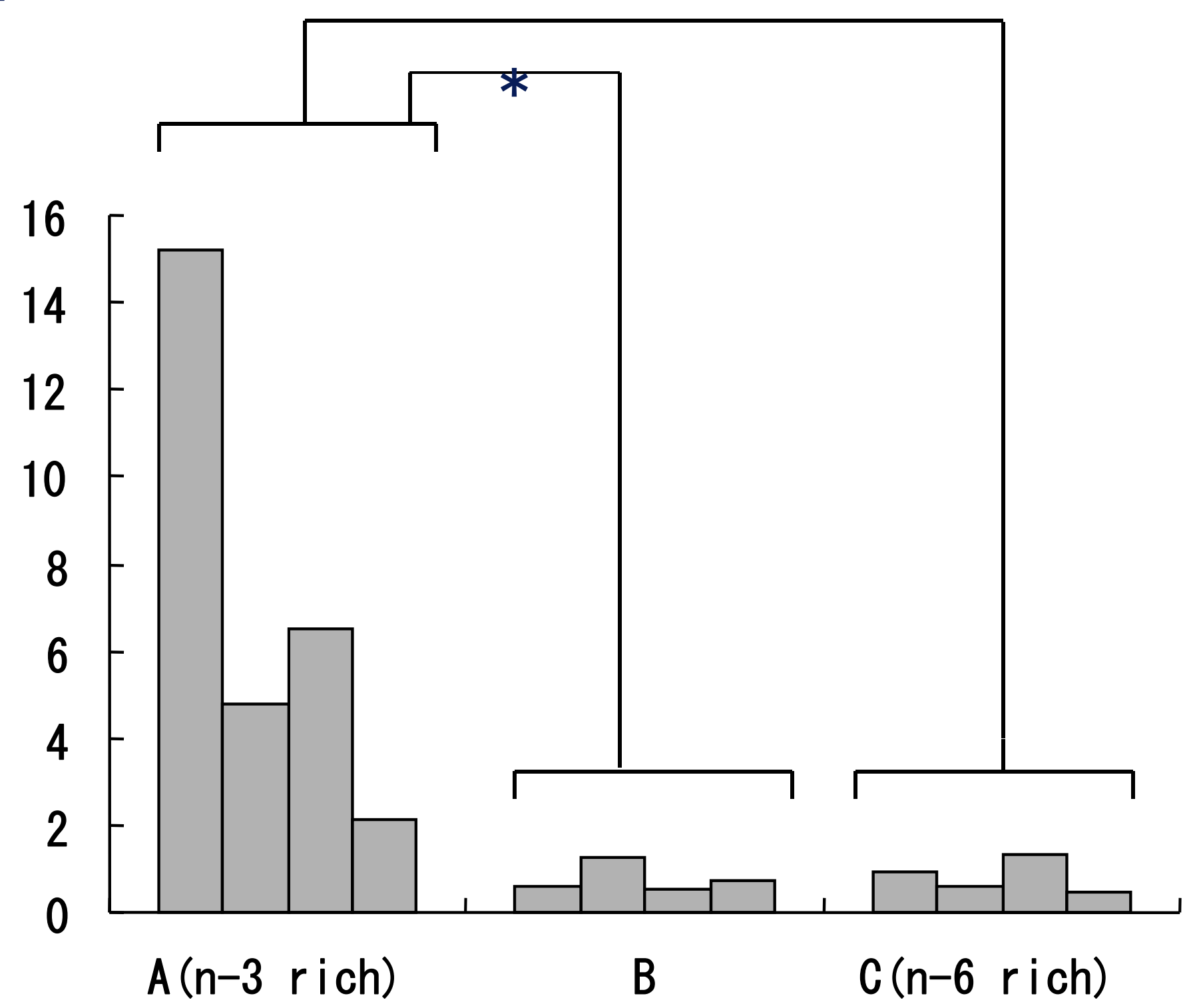


Fig. 2
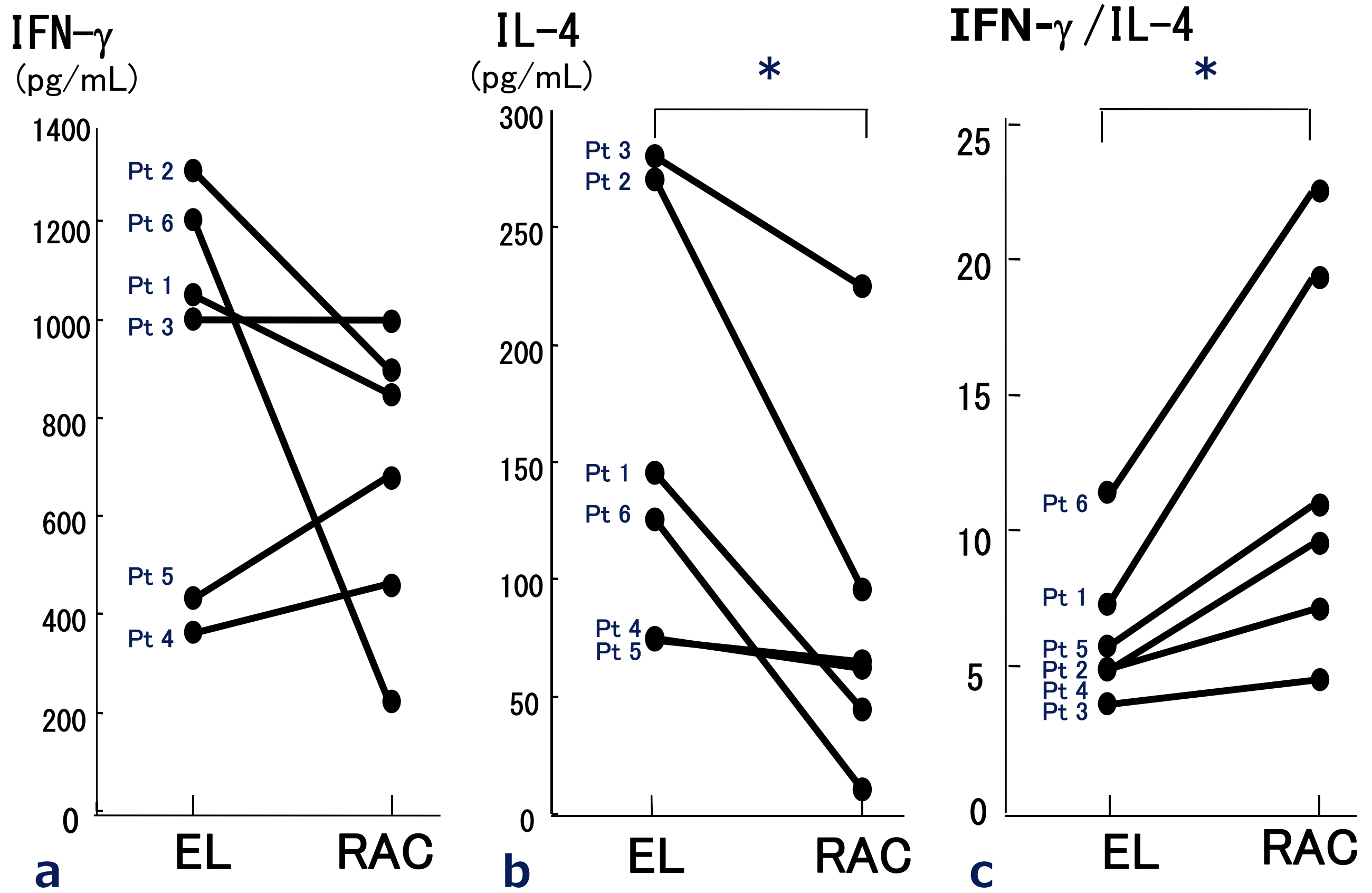

IFN- $\gamma /$ IL-4 